\title{
Optimization Study on Graphene-Coated Microfiber Bragg Grating Structures for Ammonia Gas Sensing
}

\author{
Anqi ZHANG, Yu WU*, Baicheng YAO, and Yuan GONG \\ Key Laboratory of Optical Fiber Sensing and Communications (Ministry of Education of China), University of \\ Electronic Science and Technology of China, Chengdu, 611731, China \\ ${ }^{*}$ Corresponding author: Yu WUＥ-mail: wuyuzju08@gmail.com
}

\begin{abstract}
The excitation of the surface field and evanescent enhancement in the graphene have shown sensitive to the refractive index of surrounding media and potential applications in high-sensitivity biochemical sensing. In this paper, we investigate the graphene-coated microfiber Bragg gratings (GMFBGs) with different diameters for ammonia gas sensing. The maximum sensitivity with $6 \mathrm{pm} / \mathrm{ppm}$ was achieved experimentally when the microfiber's diameter was about $10 \mu \mathrm{m}$. Moreover, by adjusting the diameter of the GMFBG, the sensing performance of the GMFBGs could be optimized. Experimental results indicated that GMFBGs with the diameter of $8 \mu \mathrm{m}-12 \mu \mathrm{m}$ would show the characteristics of the high sensitivity, relative low attenuation, and large dynamic range.
\end{abstract}

Keywords: FBG, graphene, ammonia gas sensing, structure optimization

Citation: Anqi ZHANG, Yu WU, Baicheng YAO, and Yuan GONG, "Optimization Study on Graphene-Coated Microfiber Bragg Grating Structures for Ammonia Gas Sensing," Photonic Sensors, 2015, 5(1): 84-90.

\section{Introduction}

As a new kind of material, graphene with unique electronic and optical characteristics, has attracted worldwide attention in the decade. Graphene, composed of a two-dimensional (2D) layer of carbon atoms in the honeycomb crystal lattice, has surprisingly high electron mobility and electrical conductivity due to its unique two-dimensional $\mathrm{sp}^{2}$-bonded structure [1-4]. Based on these excellent properties, new researches have been attracted on integrating graphene into potential electronic and optical devices. Sensing devices are widely studied with great results because graphene's 2D crystal structure allows every carbon atom a surface structure, providing the greatest possible specific surface area [4-9]. Large specific surface area makes graphene highly sensitive to the change in the reflective index (RI) in the local environment. The graphene based sensor for individual gas sensing has been demonstrated. The carrier concentration in graphene will be changed for the adsorption or desorption of gas molecules that act as donors or acceptors on the graphene surface [10-13]. Thus, graphene shows a great potential for bio-chemical sensing applications.

The microfiber Bragg grating (MFBG) combines the benefits of evanescent field transmission as well as the wavelength sensitivity [14]. Ordinary FBGs cannot be used for evanescent field sensing because the fundamental core mode is hardly influenced by the surrounding media [15-17]. For MFBGs, the

Received: 12 August 2014 / Revised version: 5 September 2014

(C) The Author(s) 2014. This article is published with open access at Springerlink.com DOI: $10.1007 / \mathrm{s} 13320-014-0216-\mathrm{x}$

Article type: Regular 
surrounding media act as the cladding, guiding the evanescent field to propagate along the fiber. Then, the effective refractive index (ERI) of MFBGs will be significantly affected by the external media, which will cause the shift in the Bragg wavelength.

In this work, a single layer graphene film was wrapped onto the surface of the MFBG, shown in Fig. 1, to enhance the evanescent field and improve the sensitivity to the local surrounding media. Through measuring the Bragg wavelength shift, small gas concentration changes can be detected.

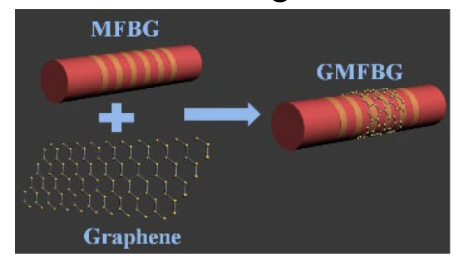

Fig. 1 Structure schematic of the GMFBG.

In order to investigate the sensing ability with the structure parameters of the graphene-coated microfiber Bragg grating (GMFBG), different diameters of GMFBGs have been studied theoretically and experimentally.

According to the theory of the Bragg grating, the Bragg wavelength $\lambda_{B}$ is

$$
\lambda_{B}=2 n_{\text {eff }} \Lambda
$$

where $n_{\text {eff }}$ and $\Lambda$ represent the effective refractive index and the period of the MFBG. When $\Lambda$ remains unchanged, $\lambda_{B}$ can change with $n_{\text {eff }}$ linearly. In the single-mode fiber, $n_{\text {eff }}$ of the basic mode can be expressed as

$$
n_{\text {eff }}^{2}=n_{1}^{2}-\left(\frac{U}{V}\right)^{2}\left(n_{1}^{2}-n_{2}^{2}\right)
$$

where $U=r \sqrt{k_{0}^{2} n_{1}^{2}-\beta^{2}}$ and $V=k_{0} r \sqrt{n_{1}^{2}-n_{2}^{2}}$ are the transverse propagation constant and normalized frequency. $r, n_{1}, n_{2}, k_{0}$, and $\beta$ represent the core radius, RI of the fiber core, RI of the cladding, the propagation constant, and phase factor, respectively.

Besides, $U$ and $V$ satisfy the following equations:

$$
\left\{\begin{array}{l}
\frac{U J_{m-1}(U)}{J_{m}(U)}=-\frac{W K_{m-1}(W)}{K_{m}(W)} \\
U^{2}+W^{2}=V^{2}
\end{array} .\right.
$$

Using the approximation for the Gauss field and method of finite element analysis to simplify the equations, the relationship between $U$ and $V$ can be expressed as $[18,19]$

$$
U=-\frac{(1+\sqrt{2}) V}{1+\left(4+V^{4}\right)^{\frac{1}{4}}} .
$$

Plugging (4) into (2), $n_{\text {eff }}$ can be obtained:

$$
\begin{aligned}
& n_{\mathrm{eff}}^{2}=n_{1}^{2}-\left(\frac{1+\sqrt{2}}{1+\left(4+V^{4}\right)^{\frac{1}{4}}}\right)^{2}\left(n_{1}^{2}-n_{2}^{2}\right) \\
& =n_{1}^{2}-\left\{\frac{1+\sqrt{2}}{1+\left[4+4 k_{0}^{4} r^{4}\left(n_{1}^{2}-n_{2}^{2}\right)^{2}\right]^{\frac{1}{4}}}\right\}^{2}\left(n_{1}^{2}-n_{2}^{2}\right) .
\end{aligned}
$$

In fact, $n_{1}$ and $k_{0}$ are constants. It is easy to see that $n_{\text {eff }}$ is a function of $r$ and $n_{2}$. Combining with (1), $\lambda_{B}$ changes with the radius and RI of the cladding [20].

Meanwhile, we define a sensitivity parameter $K$ to describe the sensitivity of the gas sensor:

$$
K=\frac{\partial \lambda_{B}}{\partial n_{\text {eff }}} .
$$

According to (5), the core radius $r$ and RI of the cladding $n_{2}$ are two factors that determine $n_{\text {eff. }}$. So $K$ depends on the changes in $r$ and $n_{2}$. Thus for the gas sensing applications, it can be observed that some nonlinear relationship exists between the sensitivity and diameter of the MFBG and GMFBG.

The electric field distributions of MFBGs and GMFBGs are simulated using the finite element method. According to the simulated results in Fig.2, with the same diameter, the coated graphene enhances the evanescent fields of MFBGs significantly, and more energy is radiated into the surrounding media. For the GMFBGs, with the same coated length of graphene in Figs.2(d), 2(e), and 2(f), when the diameter decreases, larger proportion of transmission light propagates outside the fiber core as evanescent fields, providing a good opportunity for the interaction between the light and surrounding media. As graphene enhances the evanescent field, it can be predicted that a higher sensitivity will be 
obtained in the GMFBG than that in the MFBG.

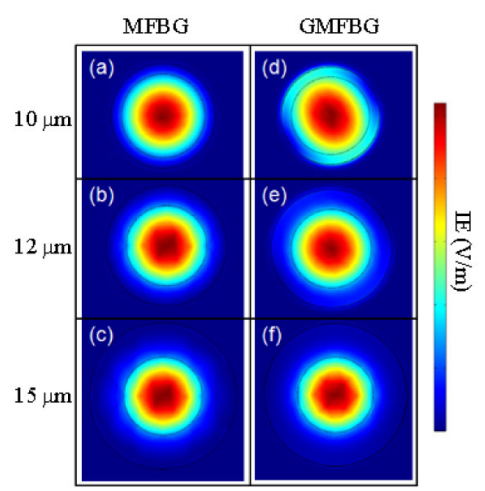

Fig. 2 Simulated results of mode field distributions of MFBGs and GMFBGs with different diameters: $\sim 10 \mu \mathrm{m}, \sim 12$ $\mu \mathrm{m}$, and $\sim 15 \mu \mathrm{m}$.

\section{Experiments}

\subsection{Fabrication for the GMFBGs}

In the experiments, FBGs were fabricated by the 248-nm KrF excimer laser with the Bragg wavelength of about $1558.3 \mathrm{~nm}$. Hydrofluoric acid was used to etch FBGs to form different diameters of MFBGs: $\sim 10 \mu \mathrm{m}, \sim 12 \mu \mathrm{m}$, and $\sim 15 \mu \mathrm{m}$. The fabrication process is shown in Fig. 3(a), and the scanning electron microscope (SEM) photographs with different diameters of MFBGs are presented in Fig. 3(b).

In this structure, the graphene film was fabricated by the chemical vapor deposition (CVD) method. Importing $\mathrm{CH}_{4}$ gas mixture into the system, the carbon atoms in a single layer would be deposited on the $\mathrm{Cu}$ foil, forming the graphene film with the 2D structure. Polymethyl methacrylate (PMMA) was then spin-coated on the surface of the graphene- $\mathrm{Cu}$ foil. To achieve a structure of PMMA-graphene, the $\mathrm{FeCl}_{3}$ solution was used to remove the $\mathrm{Cu}$ foil. After these, the graphene film could be transferred to the surface of MFBGs on the $\mathrm{MgF}_{2}$ substrate, with the coated length of about $1 \mathrm{~mm}$ in our experiment. Finally, using acetone and alcohol to clear PMMA, then the hybrid waveguide of the GMFBG was obtained. The process is shown in Fig. 3(c). The Raman spectrum of CVD grown graphene was also measured in Fig. 3 (d), showing a very weak $D$ peak and an intensity ratio $(\sim 0.4)$ of the 2D peak to the $G$ peak. The Raman spectrum confirms a high quality of our prepared graphene.
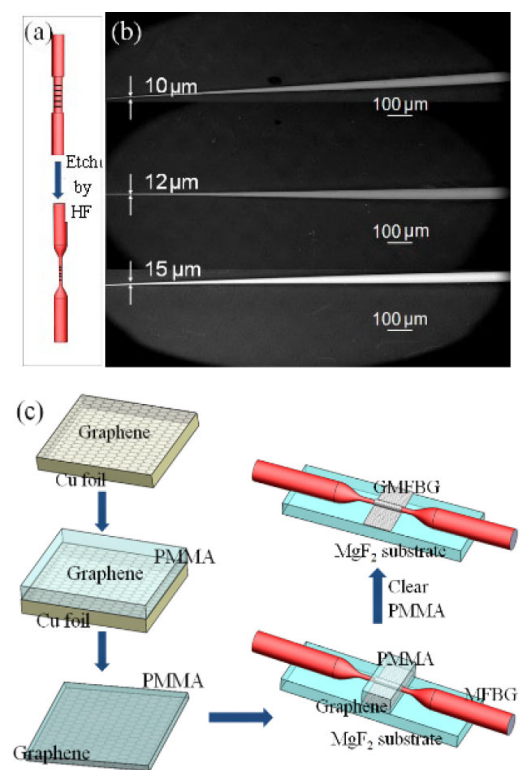

(d)

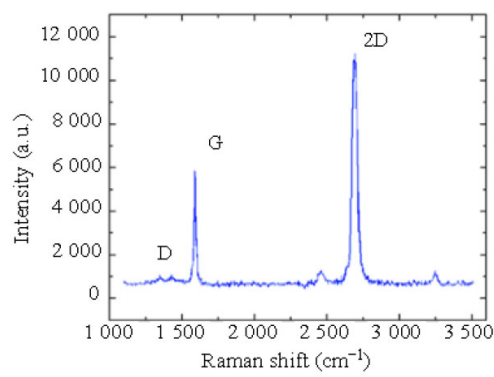

Fig. 3 Drawings of MFBG and GMFBG for (a) fabrication process of MFBG, (b) SEM photographs of MFBGs with different diameters, (c) process of making GMFBG, and (d) Raman spectrum of CVD grown graphene.

\subsection{Experimental setup}

The experimental setup for ammonia gas sensing is shown in Fig. 4. A broadband laser was adopted as



Fig. 4 Experimental setup for gas sensing. 
the light source, with the wavelength range from $1510 \mathrm{~nm}$ to $1590 \mathrm{~nm}$. Light was launched into the system via a $3-\mathrm{dB}$ coupler to a sealed gas chamber. The liquid ammonia was injected into the gas chamber away from the GMFBG sensor to minimize the thermal effect during the process of volatilize. At the output end, light signals were collected by the optical spectrum analyzer (OSA, N744A, Agilent, USA), with the maximum resolution of $0.1 \mathrm{pm}$.

Figure 5 shows the reflection spectra of the MFBGs and GMFBGs with different diameters. In accordance with the theoretical analysis, when the diameters of the MFBGs decreased, the Bragg wavelength would move toward the shorter wavelength. During etching, a decrease in intensity was also observed, which could be explained by the mismatch of the numerical aperture between the un-etched and etched fiber regions [21]. However, when the diameter decreased to $10 \mu \mathrm{m}$ or below, the reflection spectra became irregular, and some side-peaks might appear after etching, as shown in Fig. 5(a). This observation could be due to the defects introduced along the uneven grating region

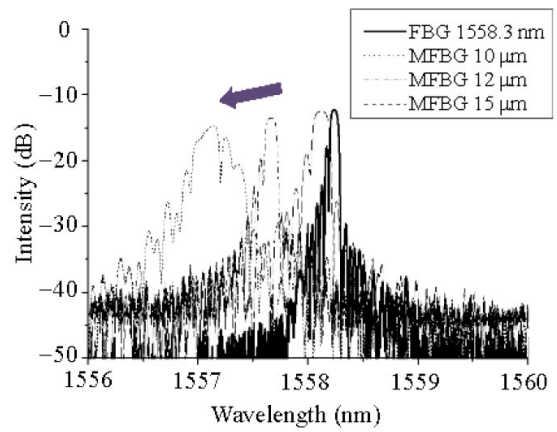

(a)

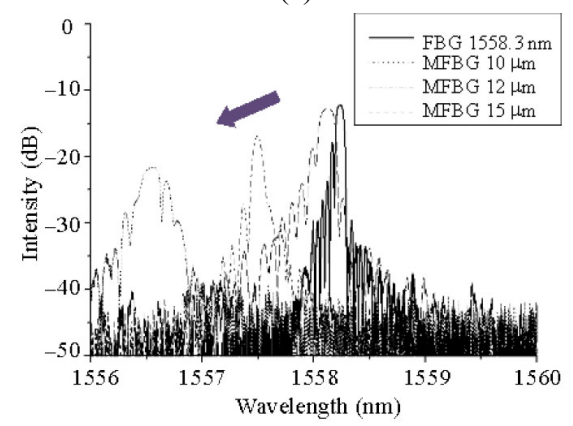

(b)

Fig. 5 Reflection spectra of (a) the MFBGs in air and (b) the GMFBGs in air.
[21]. The deterioration of the spectrum has a certain influence on the accuracy of measurement. But in this experiment, this defect could be ignored because the Bragg wavelength of GMFBGs could still be well detected by the OSA with a high resolution. Figure 5(b) presents the measured spectra of GMFBGs, with diameters of about $10 \mu \mathrm{m}, 12 \mu \mathrm{m}$, and $15 \mu \mathrm{m}$, respectively. It is noted that the coated graphene would generate both additional transmission loss and further blue shifts of GMFBGs than those of MFBGs.

\subsection{Experimental results and analysis}

Different diameters of GMFBGs were tested for $\mathrm{NH}_{3}$ gas with the concentrations of $0 \mathrm{ppm}, \sim 10 \mathrm{ppm}$, $\sim 50 \mathrm{ppm}$, and $\sim 100 \mathrm{ppm}$. The spectrum responses are shown in Figs. 6(a), 6(b), and 6(c). Under the concentration of $\sim 10 \mathrm{ppm}$, the Bragg wavelength shifts of the GMFBGs were detected to be $\sim 60 \mathrm{pm}$, $\sim-40 \mathrm{pm}$, and $\sim 0 \mathrm{pm}$ for $\sim 10 \mu \mathrm{m}, \sim 12 \mu \mathrm{m}$, and $\sim 15 \mu \mathrm{m}$, respectively. These results indicated that the GMFBG with a smaller diameter would be more sensitive to the gas concentration alteration.

According to Fig. 6, specific spectral shifts and peak intensities of GMFBGs can be drawn in Fig. 7.

Calculated from Fig. 7(a), the resolution of the GMFBGs for gas trace sensing $(0 \mathrm{ppm}-100 \mathrm{ppm})$ could be up to $6 \mathrm{pm} / \mathrm{ppm}$ of $\sim 10 \mu \mathrm{m}, 4 \mathrm{pm} / \mathrm{ppm}$ of $\sim 12 \mu \mathrm{m}$, and almost $0 \mu \mathrm{pm} / \mathrm{ppm}$ of $\sim 15 \mu \mathrm{m}$, respectively. The initial peak intensity of $\sim 10 \mu \mathrm{m}$ GMFBG was $-21.5 \mathrm{~dB}$ in our experiment, which would be difficult to be detected when it became worse. The attenuations of GMFBGs could also be calculated from Fig. 7 (b), $0.08 \mathrm{~dB} / \mathrm{ppm}$ of $\sim 10 \mu \mathrm{m}$, $0.07 \mathrm{~dB} / \mathrm{ppm}$ of $\sim 12 \mu \mathrm{m}$, and $0.03 \mathrm{~dB} / \mathrm{ppm}$ of $\sim 15 \mu \mathrm{m}$. Thus for different diameters of GMFBGs, a higher sensitivity would bring a larger attenuation and more irregularities in the reflection spectrum. To further demonstrate the enhancement effect by graphene, MFBGs with different diameters were also tested for $\mathrm{NH}_{3}$ gas sensing, shown in Fig. 7. However, it is obvious that MFBGs without the graphene cladding were almost not sensitive to gas adsorption. The 
maximum spectral shift induced by $\mathrm{NH}_{3}$ for the MFBG was smaller than $0.2 \mathrm{pm} / \mathrm{ppm}$.

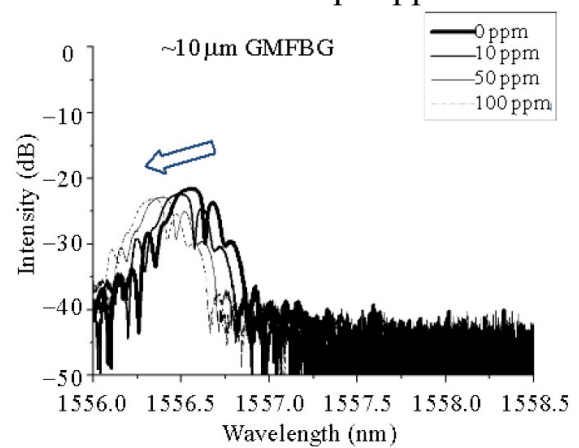

(a)

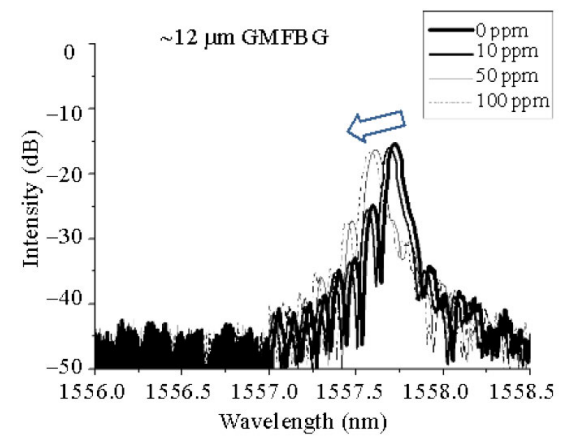

(b)

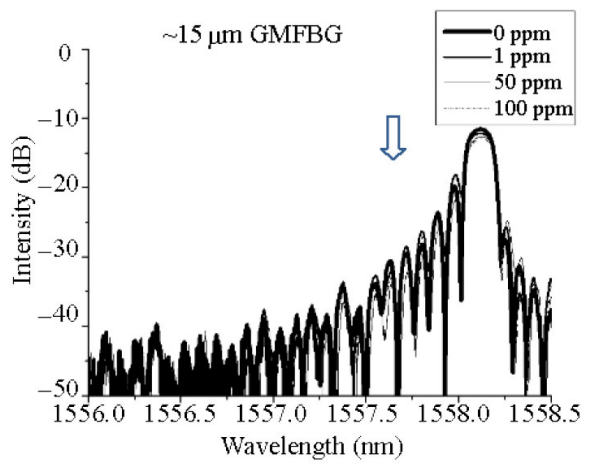

(c)

Fig. 6 In different concentrations of $\mathrm{NH}_{3}$, the reflection spectra of (a) GMFBG with the diameter of $\sim 10 \mu \mathrm{m}$, (b) GMFBG with the diameter of $\sim 12 \mu \mathrm{m}$, and (c) GMFBG with the diameter of $\sim 15 \mu \mathrm{m}$.

In principle, $\mathrm{NH}_{3}$ is an electron donor to graphene, the gas adsorption will increase the number of electrons on the surface of graphene, changing the local carrier concentration, hence changing the dielectric constant of the graphene film [13]. It appears that both the real and imaginary parts of the dielectric constant change with the gas concentration, which is responsible for more obvious shift in the wavelength and attenuation of intensity than those of the MFBG only.

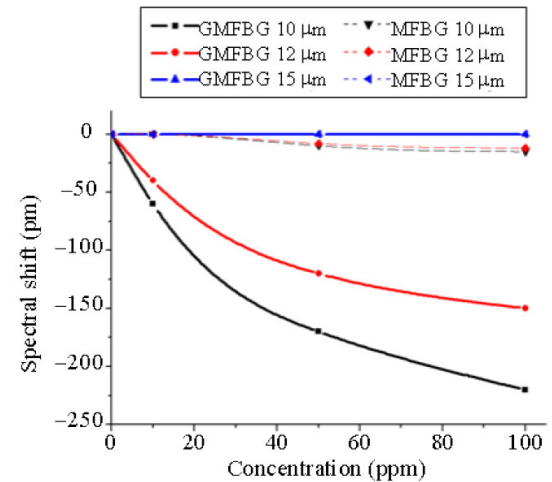

(a)

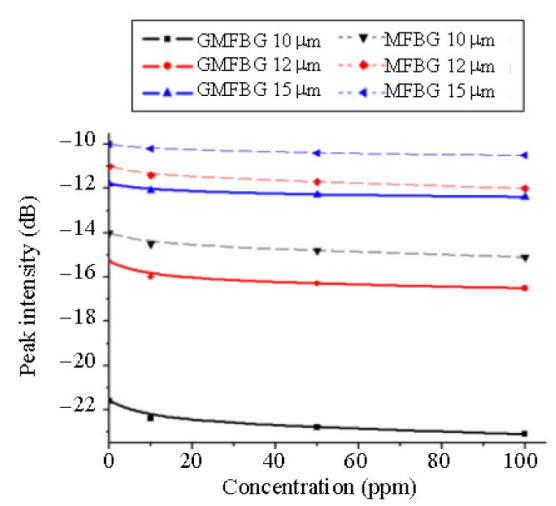

(b)

Fig. 7 For different concentrations of $\mathrm{NH}_{3}$ gas sensing: (a) spectral shifts of the GMFBGs and MFBGs and (b) peak intensities of the GMFBGs and MFBGs.

Based on different diameters of GMFBGs, the sensitivity and attenuation could be curve-fitted, as shown in Figs.8(a) and 8(b). From the spectral shift and peak intensity of GMFBGs in Fig. 7, we can estimate the maximum gas concentration to be detected for different diameters, $\sim 150 \mathrm{ppm}$ of $\sim 10 \mu \mathrm{m}, \sim 120 \mathrm{ppm}$ of $\sim 12 \mu \mathrm{m}$, and $\sim 20 \mathrm{ppm}$ of $\sim 15 \mu \mathrm{m}$, respectively, and the curve-fitting results are shown in Fig. 8(c). For the three parameters, when the diameter increases, both the sensitivity and the attenuation decrease, forming a smaller dynamic range of the gas concentration. Thus, the diameter should be optimized to obtain the best performance of the GMFBG gas sensor.

Here, we define a performance parameter $H$, which can be used to evaluate the comprehensive performance of the gas sensor. For this sensing structure, in order to get the higher sensitivity, lower attenuation, and larger dynamic range, $H$ can be expressed by the following equation: 


$$
H=\left[\frac{\ln S \times \ln D^{\prime}}{A}+C_{1}\right] / C_{2}
$$

where $S, D^{\prime}$, and $A$ represent the sensitivity, dynamic range of the gas concentration, and attenuation, respectively. According to the experimental results and fitting data, we choose the constants $C_{1}=70$ and $C_{2}=200$ to ensure $H>0$ and make $H$ normalized.

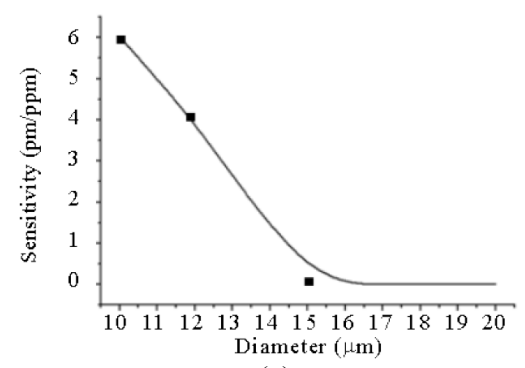

(a)

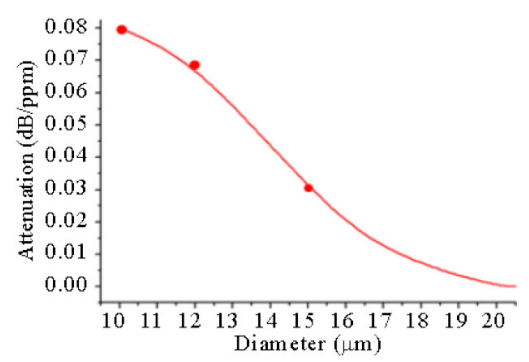

(b)

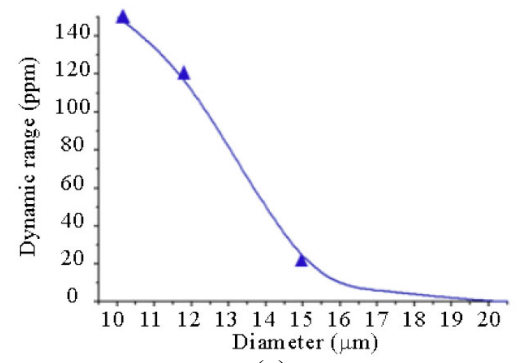

(c)

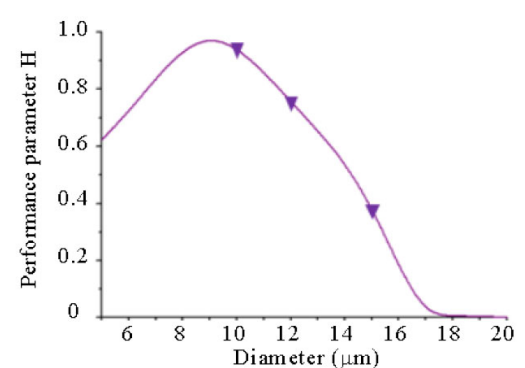

(d)

Fig. 8 Fitted curves of (a) sensitivity for different diameters of GMFBGs, (b) attenuation for different diameters of GMFBGs, (c) dynamic range of the gas concentration for different diameters of GMFBGs, and (d) performance parameter $H$ for different diameters of GMFBGs.
Combined with the fitting equations of $S, D^{\prime}$, and $A$, the fitted curve of $H$ with the changing diameter $D$ can be drawn in Fig. 8(d). It can be observed that, when the diameter is $\sim 9 \mu \mathrm{m}$, we can achieve the maximum $H$, which indicates that the GMFBG with a diameter of $\sim 9 \mu \mathrm{m}$ will show the best performance. Within the range of $6 \mu \mathrm{m}-12 \mu \mathrm{m}$, the performance of the GMFBG is better. Concerning the actual situation, the GMFBG under $\sim 8 \mu \mathrm{m}$ will get poor operability and more defects. Therefore, when the diameter is within $8 \mu \mathrm{m}-12 \mu \mathrm{m}$, the GMFBG sensor shows the good performance with the high sensitivity, low attenuation, and large dynamic range.

\section{Conclusions}

In conclusion, we investigated the graphenecoated microfiber Bragg grating structure for $\mathrm{NH}_{3}$ gas sensing. Theoretical analyses and experimental results demonstrated that the graphene coated film enhanced the evanescent field on the microfiber FBG for chemical gas sensing, and a higher sensitivity could be achieved with a smaller diameter of the GMFBG. However, the balance between the sensitivity and attenuation should be considered for this hybrid structure. Within the range of $8 \mu \mathrm{m}-12 \mu \mathrm{m}$, the sensing structure would show the good performance in gas sensing. Besides, this kind of GMFBG gas sensor also shows good properties in reversibility and repeatability for $\mathrm{NH}_{3}$ sensing, and it also has additional advantages such as the probe miniaturization, low cost, capability of online monitoring and remote sensing.

\section{Acknowledgment}

The authors greatly thank Dr. Zegao Wang and Prof. Yuanfu Chen of the State Key Lab of Electronic Thin Films and Integrated Devices, University of Electronic Science \& Technology of China, for their help on the preparation and test of the graphene used in the study and useful discussions. This work was supported by the 
National Natural Science Foundation of China under Grant 61290312, 61107072, and 61107073. It was also supported by Program for Changjiang Scholars and Innovative Research Team in Universities of China (PCSIRT) and the "111 Project" of China Education Ministry.

Open Access This article is distributed under the terms of the Creative Commons Attribution License which permits any use, distribution, and reproduction in any medium, provided the original author(s) and source are credited.

\section{References}

[1] F. Bonaccorso, Z. Sun, T. Hasan, and A. Ferrari, "Graphene photonics and optoelectronics," Nature Photonics, 2010, 4(9): 611-622.

[2] J. A. Kim, T. Hwang, S. R. Dugasani, R. Amin, A. Kulkarni, S. H. Park, et al., "Graphene based fiber optic surface plasmon resonance for bio-chemical sensor applications," Sensors and Actuators B: Chemical, 2013, 187(SI): 426-433.

[3] G. Ko, H. Y. Kim, J. Ahn, Y. M. Park, K. Y. Lee, and J. Kim, "Graphene-based nitrogen dioxide gas sensors," Current Applied Physics, 2010, 10(4): 1002-1004.

[4] F. Yavari and N. Koratkar, "Graphene-based chemical sensors," The Journal of Physical Chemistry Letters, 2012, 3(13): 1746-1753.

[5] W. Tan, M. Hofmann, Y. Hsieh, M. Lu, and Y. Chen, "A graphene-based surface plasmon sensor," Nano Research, 2012, 5(10): 695-702.

[6] H. Li, Y. Anugrah, J. K. Steven, and M. Li, “Optical absorption in graphene integrated on silicon waveguides," Applied Physics Letters, 2012, 101(11): 111110-1-111110-3.

[7] S. Thongrattanasiri, F. H. L. Koppens, and F. J. G. Abajo, "Complete optical absorption in periodically patterned graphene," Physical Review Letters, 2012, 108(4): 047401-1-047401-5.

[8] B. Yao, Y. Wu, Y. Gong, and Y. Rao. "A highly sensitive and fast response molecular sensor based on graphene coated microfiber," in Proc. SPIE, vol. 8421, pp. 861-864, 2012.

[9] F. Schedin, A. K. Geim, S. V. Morozov, E. W. Hill, P. Blake, M. I. Katsnelson, et al., "Detection of individual gas molecules adsorbed on graphene,' Nature Materials, 2007, 6(9): 652-655.

[10] I. F. Rivera, R. K. Joshi, and J. Wang, "Graphene-based ultra-sensitive gas sensors," in IEEE Sensors 2010 Conference, pp. 1534-1537, 2010.

[11] B. Yao, Y. Wu, Y. Cheng, X. Liu, Y. Gong, and Y. Rao, "Graphene based microfiber gas sensor," in Proc. SPIE, vol. 8421, pp. 8421CD1-CD4, 2012.

[12] S. Some, Y. Xu, Y. Kim, Y. Yoon, H. Qin, A. Kulkarni, et al., "Highly sensitive and selective gas sensor using hydrophilic and hydrophobic graphenes," Scientific Reports, 2013, 3(1868): 1-4.

[13] Y. Wu, B. C. Yao, Y. Cheng, X. Liu, Y. Gong, and Y. Rao, "Hybrid graphene-microfiber waveguide for chemical gas sensing," IEEE Journal of Selected Topics in Quantum Electronics, 2014, 20(1): 4400206.

[14] R. Liang and Q. Sun, "Investigation on micro/nanofiber Bragg grating for refractive index sensing," Optics Communications, 2012, 285(6): $1128-1133$.

[15] R. Liang, Q. Sun, J. Wo, and D. Liu, "Theoretical investigation on refractive index sensor based on Bragg grating in micro/nanofiber," Acta Physica Sinica, 2011, 60(10): 390-394.

[16] Y. Wu, B. Yao, A. Zhang, Y. Rao, Z. Wang, Y. Cheng, et al., "Graphene-coated microfiber Bragg grating for high sensitivity gas sensing," Optics Letters, 2014, 39(5): 1235-1237.

[17] A. Zhang, S. Gao, G. Yan, and Y. Bai, "Advances in optical fiber Bragg grating sensor technologies," Photonic Sensors, 2012, 2(1): 1-13.

[18] Y. Liu, F. Che, Z. Jia, H. Fu, H. Wang, and M. Shao, "Investigation on the characteristics of micro/nanofiber Bragg grating for refractive index sensing," Acta Physica Sinica, 2013, 62(10): 104218.

[19] Y. Liao, Fiber optics - principles and applications. Beijing: Tsinghua University Publishing House, 2010: 358-366.

[20] D. Li, Y. Gong, and Y. Wu, "Tilted fiber Bragg grating in graded-index multimode fiber and its sensing characteristics," Photonic Sensors, 2013, 3(2): 112-117.

[21] A. Cusano, D. Paladino, A. Cutolo, A. Iadicicco, and S. Campopiano, Fiber Bragg grating sensors: recent advancements, industrial applications and market exploitation. Netherlands: Bentham Science Publishers Ltd., 2011: 238-269. 